\title{
PENICILLIN IN THE TREATMENT OF NEONATAL SEPSIS
}

\author{
BY \\ F. M. B. ALLEN, M.D., F.R.C.P., J. EDGAR MORISON, M.D., B.Sc., and \\ W. R. RUTHERFORD, M.B., B.Ch.
}

(From the Departments of Paediatrics and Pathology, Queen's University, and the Belfast City Hospital, on behalf of The Belfast Penicillin Clinical Trials Committee)

The object of this research has been to determine the place of penicillin in the treatment of neonatal sepsis. It is well known that in the neonatal period only a minority of cases present symptoms which enable an exact diagnosis to be made either of the site, or sites, of infection or of the nature of the infecting agent. Many of these patients present a distinctive clinical picture, however, and have an illness of sudden onset with some or all of the following symptoms: fretfulness or apathy, refusal to feed, diarrhoea, vomiting, and an abnormal fall of the post-natal birth weight, or a downward deflection of the weight curve if this has begun to climb. The temperature may be raised at some period of the illness, but absence of fever will not exclude the presence of infection. The respiration rate may be increased and icterus may seem more pronounced than would be expected in physiological jaundice of the newborn. The condition sometimes assumes epidemic proportions in maternity hospitals. When diarrhoea predominates there is then a tendency to label it epidemic diarrhoea of the newborn and to regard it, sometimes with little further investigation, as a primary gastro-enteritis of unknown etiology.

Careful and detailed autopsies on cases of neonatal sepsis, especially when dehydration has been in some measure controlled, will very rarely show any lesions in the intestinal tract, but will often show evidence of infection elsewhere, especially in the respiratory tract. Histological examination will frequently reveal acute inflammatory reaction in the nose, larynx and pharynx, lungs and nearly always in the middle ears. Some cases may show only thrombi in intracranial or renal veins, and it is then impossible either to implicate or to exclude a low-grade and disseminated bacterial infection. It is not useful at the moment to discuss whether symptoms, which in many of these cases might suggest a primary gastro-enteritis, result from a primary invasion by viruses, bacteria or both pathogenic agents in symbiosis through the respiratory tract or other portal of entry. It is enough to emphasize that, if dehydration is controlled, bacterial infection, especially of the respiratory tract, be it primary or secondary, plays a very important rôle and often determines the fatal outcome.

While the effect of penicillin must also be assessed in cases where the diagnosis can be more fully established, the main object of the present investigation has been to study its influence on the above type of case on an admittedly empirical basis. The cases treated may have been produced by different viruses and bacteria and their evolution was probably influenced by secondary microbic invasion. Such secondary infection may result from cross-infection from other infants, or from mothers or attendants, in spite of the segregation of infants in small nurseries and a careful nursing technique; it may also be due to organisms which are normally saprophytic on the skin or epithelial-lined passages of the infant. A careful and detailed clinical and autopsy study may indicate something of the various factors contributing to death, but there is no hope at present of making any scientifically satisfactory, or even clinically useful, subdivision of the majority of these cases.

\section{Selection of Clinical Material}

Eighty-nine cases, all within the first month of life, have been included in this survey. Eighteen cases were treated with penicillin, chiefly during the early part of the study, on an individualized basis and without collateral controls, and these will only be referred to later. The other seventy-one cases were treated on a definite plan and thirty-five received penicillin and sulphadiazine and thirty-six sulphadiazine only. Infants in the neonatal department of the Jubilee Maternity Department of the Belfast City Hospital were kept under specially close supervision. Any refusal to feed, the onset of diarrhoea or vomiting, abnormal loss of weight, rise of temperature, increase in respiration rate, or any sign of toxicity was at once investigated and the infant transferred to a sick nursery under a staff specially trained and instructed in the procedures of this investigation. A detailed physical examination was made, but blood cultures or catheter specimens of urine were not examined in this series. In the initial stages blood cultures are unlikely to be helpful because even three or more cultures are often negative and the significance of many of the 
organisms recovered may be questioned even when the most careful technique is employed. Treatment cannot be withheld until repeated cultures have been taken. The decision as to whether the baby was infected or not was therefore made on the clinical evidence alone. Alternative cases of sepsis were assigned either to receive sulphadiazine therapy in the control group or to receive, in addition, penicillin therapy in the test group. Cases were treated until they recovered or died.

Three cases receiving penicillin and one receiving sulphadiazine only died within twenty-four hours, and one test and two control cases were taken home against advice while the outcome was still in doubt. One case in each group was shown at autopsy to have thrombotic lesions much older than the period of chemotherapy, and one control case was found at autopsy to have a developmental defect (case 2; Morison, 1945). It has been thought right to exclude these five test group and five control group cases. In this series there are therefore sixty-one cases available for analysis. Owing to the number and severity of the cases encountered the hospital was at one period closed and thoroughly cleansed and disinfected. It was a definite clinical impression that the nineteen cases treated after this showed, when they were compared with the first forty-two cases treated, less marked intestinal symptoms and more obvious involvement of the respiratory system. It has been thought useful to distinguish these two groups and to give the results from each separately, followed by the total (e.g. $9+5=14$ ).

The birth weights and the weights at onset of illness show a rather wide range of values. In Table 1 the means of these values are given after the cases have been divided according to treatment and outcome. The mean ages at onset are also given, but here an accumulation of values a little below the mean and a few far above it made the calculation of figures for the standard deviations of no consequence. On analysis, however, the groups were very similar in age composition. The mean weight is lowest in the group dying after treatment with penicillin and sulphadiazine. This is not statistically significant and it was due to an unfortunate, but entirely unintentional, inclusion of all six infants of less than $5 \frac{1}{2} \mathrm{lb}$. in the test group. Four of these premature infants died. It has been thought necessary, therefore, to give additional figures to show the effect of exclusion of all these premature infants.

\section{Therapeutic Procedures}

It was not thought justifiable to withhold from either group the possible benefit of sulphadiazine, especially in view of the frequency of intestinal symptoms. Sulphadiazine has been found valuable not only in combating possible enteral pathogens, but also for its action on parenteral infections, the toxins of which are probably very often responsible for diarrhoea. The control group, therefore, received $0.25 \mathrm{gm}$. of sulphadiazine every three hours and the test group in addition had 1500 units of penicillin in $\frac{1}{2}$ c.c. sterile distilled water given by intramuscular injection with a fine needle every three hours. This represented in nearly every case well over 1500 units per pound of body weight per twenty-four hours and, judged on this basis, on the reports of other workers and on a few observations made here, should have produced very satisfactory bacteriostatic levels. Control of dehydration and feeding was similar in both groups. Oral thrush infection was controlled by 2 per cent. aqueous solution of gentian violet.

\section{Results}

Control Series. The results are presented in Table 2. It will be seen that fourteen controls (sulphadiazine treated) lived and seventeen died, and that thirteen treated (sulphadiazine and penicillin treated) cases lived and seventeen died. If premature infants are excluded there are still fourteen controls who lived and seventeen who died, while eleven treated cases lived and thirteen died. It is evident, even without statistical analysis, that these figures hold out no hope of demonstrating any difference, if such exists, between the two groups with a number of cases which there is any immediate likelihood of obtaining, even by a large-scale investigation.

When recovery or death occurred within two or three days it may be suggested that the whole infection was due to a virus, and that only when the illness was prolonged beyond this period could penicillin exert an influence on the secondary bacterial flora. The data given in Table 2 allows the exclusion of cases whose outcome was determined by the second or third day. Even after this very questionable procedure the results, admittedly on undesirably smaller groups, provide no evidence which, on statistical analysis, favours penicillin therapy.

Further information might be obtained by a study of the duration and severity of illness in the two groups. In all but nine cases daily observations of the body weight were made. In cases which recovered, the number of days of illness before the downward movement of the weight curve was reversed probably measures the duration of the illness as well as any subjective clinical impression.

TABLE 1

MEAN WEIGHT AND AGE OF INFANTS IN THE VARIOUS TREATMENT GROUPS

\begin{tabular}{|c|c|c|c|c|c|c|c|}
\hline & & \multirow{2}{*}{\multicolumn{2}{|c|}{ Sulphadiazine }} & \multicolumn{4}{|c|}{ Penicillin + Sulphadiazine } \\
\hline & & & & \multicolumn{2}{|c|}{ All cases } & \multicolumn{2}{|c|}{ Prematures excluded } \\
\hline & & Lived & Died & Lived & Died & Lived & Died \\
\hline $\begin{array}{l}\text { Birth weight (lb.) } \\
\text { Onset weight (lb.) } \\
\text { Mean age at onset (days) }\end{array}$ & $\begin{array}{l}\cdots \\
\cdots \\
\cdots\end{array}$ & $\begin{array}{c}7 \cdot 38 \pm 1 \cdot 06 \\
6 \cdot 56 \pm 0 \cdot 97 \\
6 \cdot 1\end{array}$ & $\begin{array}{c}7 \cdot 51 \pm 0.84 \\
6.63 \pm 0 \cdot 87 \\
4 \cdot 9\end{array}$ & $\begin{array}{c}7 \cdot 40 \pm 1 \cdot 07 \\
6 \cdot 78 \pm 1 \cdot 66 \\
7 \cdot 7\end{array}$ & $\begin{array}{c}6 \cdot 38 \pm 1 \cdot 38 \\
5 \cdot 71 \pm 1 \cdot 31 \\
6 \cdot 0\end{array}$ & $\begin{array}{c}7 \cdot 93 \pm 0 \cdot 98 \\
7 \cdot 55 \pm 1 \cdot 23 \\
8 \cdot 0\end{array}$ & $\begin{array}{c}7 \cdot 17 \pm 0 \cdot 80 \\
6 \cdot 34 \pm 0 \cdot 78 \\
5 \cdot 0\end{array}$ \\
\hline
\end{tabular}


TABLE 2

NUMBER OF PATIENTS RECOVERING AND DYING AND DURATION OF ILLNESS AND LOSS OF WEIGHT

\begin{tabular}{|c|c|c|c|c|c|c|}
\hline & & & & \multirow{2}{*}{$\begin{array}{l}\text { Sulphadiazine alone } \\
\text { (No prematures) }\end{array}$} & \multicolumn{2}{|c|}{ Penicillin + sulphadiazine } \\
\hline & & & & & All cases & Prematures excluded \\
\hline \multicolumn{4}{|c|}{ RECOVERY } & $9+5=14$ & $8+5=13$ & $8+3=11$ \\
\hline \multicolumn{4}{|c|}{$\begin{array}{l}\text { Total .. } \\
\text { Weight fall reversed within: }\end{array}$} & & & \\
\hline 2 days & .. & .. & .. & $5+1=6$ & $2+4=6$ & $2+3=5$ \\
\hline 3 days & . & .. & .. & $7+2=9$ & $4+4=8$ & $4+3=7$ \\
\hline $\begin{array}{r}4 \text { days } \\
\text { Weight loss }\end{array}$ & & .. & .. & $9+5=14$ & $5+4=9$ & $5+3=8$ \\
\hline \multicolumn{4}{|c|}{ Weight loss under: } & $4+3=7$ & $4+4=8$ & $4+3=7$ \\
\hline \multirow{2}{*}{$\begin{array}{r}8 \mathrm{oz} . \\
\text { over } 8 \mathrm{oz}\end{array}$} & .. & .. & .. & $8+5=13$ & $6+5=11$ & $6+3=9$ \\
\hline & .. & .. & .. & & 2 & \\
\hline \multicolumn{4}{|c|}{ DEATH } & & & \\
\hline \multicolumn{2}{|c|}{$\begin{array}{r}\text { Total } \\
\text { Death within: }\end{array}$} & . & $\because$ & $13+4=17$ & $12+5=17$ & $9+4=13$ \\
\hline 2 days & .. & .. & .. & $0+1=1$ & $6+1=7$ & $4+0=4$ \\
\hline 3 days & . & .. & .. & $4+2=6$ & $7+3=10$ & $5+2=7$ \\
\hline 4 days & . & . & . & $9+4=13$ & $9+3=12$ & $7+2=9$ \\
\hline $\begin{array}{l}5 \text { days } \\
5 \text { dave }\end{array}$ & .. & .. & .. & $11+4=15$ & $10+3=13$ & $8+2=10$ \\
\hline $\begin{array}{l}\text { after } 5 \text { days } \\
\text { Total weight }\end{array}$ & ss: & .. & .. & 2 & $2+2=4$ & $1+2=3$ \\
\hline under $4 \mathrm{oz}$ & .. & .. & .. & 1 & 3 & 2 \\
\hline $8 \mathrm{oz}$. & . & .. & . & 3 & 8 & 5 \\
\hline $12 \mathrm{oz}$. & .. & . & . & 8 & & 8 \\
\hline $16 \mathrm{oz}$ & .. & .. & .. & 10 & 11 & 8 \\
\hline over $16 \mathrm{oz}$. & .. & . & . & 3 & 1 & 1 \\
\hline
\end{tabular}

The actual loss of weight is probably less useful as many cases became ill during the period of physiological weight loss. In cases which died a prolongation of the period of illness or a reduction of the weight loss might suggest some benefit. The wide scatter of these values and the small numbers in each class have made statistical analysis impossible. This data has, however, been tabulated in Table 2. Treated and control cases show no constant or significant difference and there is no indication that penicillin has been of any benefit.

Individualized therapy. Four cases treated in the Jubilee Maternity Hospital yielded little information and neither penicillin nor sulphadiazine, singly or together, appeared to influence somewhat protracted, but low-grade, sepsis. Fourteen cases were treated in the Royal Maternity Hospital. In six the outcome was highly satisfactory but, in review, the indications for therapy were scarcely sufficient to justify a claim that neonatal sepsis was certainly present. Two cases died despite adequate amounts of penicillin. One of these was a 28-week premature infant of $2 \mathrm{lb} .11 \mathrm{oz}$., and here 10,000 units daily from the nineteenth to the twenty-fifth day of life failed to control a peritonitis from which only enterococci and B. coli were isolated and which was secondary to an infected umbilicus. In the other case a full-time infant died on the twelfth day from pneumonia after six days' treatment with 8000 units daily and then two days on 16,000 units daily. The premature infant received only a short preliminary course of sulphadiazine, but in the second case sulphadiazine was given throughout.

Four cases which did not respond initially to sulphadiazine are of interest, and penicillin probably contributed to the favourable outcome. One was a 32-week premature infant of $2 \mathrm{lb} .14 \mathrm{oz}$. whose blood culture on the eighth day of life, and while he was receiving sulphadiazine, gave a pure growth of Staphylococcus aureus. He was given 18,125 units in the next twenty-four hours, and the blood then gave complete stasis by Fleming's method in a dilution of $1: 64$. A further course of 13,125 units was given over three days, but he appeared entirely cured. A troublesome case of pemphigus reacted very favourably to 21,500 units given over four days. The other two cases appeared clinically to be cases of pneumonia and responded well to 1000 units three-hourly, and therapy was stopped after the fourth and fifth days.

Two cases received penicillin and sulphadiazine at the same time. One was probably a primary pneumonia. The other received 8000 units daily from the sixteenth day of life when he was fretful, appeared dehydrated and had a distended, tender abdomen, but an apparently healed umbilicus. From the twenty-fifth day of life there was an abundant discharge of pus from below the umbilicus and this contained Staphylococcus aureus. Penicillin was continued until the thirty-fifth day and contributed to the favourable outcome in this case of umbilical infection.

In this group the cases which responded to penicillin resembled somewhat those infections of later childhood or adult life which are amenable to penicillin. There was some evidence of a primary infection, presumably by an organism susceptible to penicillin, and apparently the condition of the infant, or the location of the lesion, did not favour heavy secondary infection, especially of the respiratory tract by resistant organisms.

\section{Discussion of Results}

It will be appreciated that this survey is a comparison of combined penicillin and sulphadiazine 
treatment with sulphadiazine therapy alone. At one time neonatal infections may be mild and the death rate low and at another the mortality may be high. It is, therefore, impossible to compare results from year to year. There is a clinical impression that sulphadiazine does make some contribution to the control of neonatal infections. In certain well-defined conditions such as staphylococcal infections, and probably in some cases of primary bacterial pneumonia penicillin possesses some advantage over sulphadiazine. It would seem, however, that the organisms responsible for much of the sepsis in the newborn are no more susceptible to penicillin than to sulphadiazine. It may be suggested that death is, therefore, due to a septicaemia or toxaemia originating from the bowel, where the epithelial barrier may be damaged. The severity of the intestinal disturbance is, however, little guide to the outcome, and death often occurs when the intestinal symptoms have abated. In the absence of anatomical lesions in the bowel wall, of reaction in the mesenteric lymph glands or of pathogenic intestinal bacteria, and in cases under oral treatment with a sulphonamide derivative, it would be well to look elsewhere before accepting this superficially attractive hypothesis.

The high incidence of respiratory tract infections has already been commented upon. It would be reasonable to hope, if the bacteria concerned were pathogens derived from the respiratory tract of adults, that a high proportion would be sensitive to penicillin. It is possible, however, that the organisms are derived from the normal flora of the nose and throat of the newborn, and that toxins acting on immature structures, associated virus infection or some other factors, depress the normal defence mechanisms which include the protective reflexes guarding the respiratory tract. Torrey and Reese (1944, 1945) have reviewed the somewhat conflicting reports on the initial aerobic flora of the newborn. They confirm the observation of Kneeland (1930) that many potential pathogens of the adult respiratory tract show little tendency to persist or grow out in the nasopharynx of the newborn infant. But, since Staphylococcus aureus was present in almost 90 per cent. of sixty-three infants by the end of the first week, it is unlikely to be absent from lesions caused by any extension of organisms from the nose or throat. Though Kneeland and Torrey and Reese have reported a lower incidence of adaptation of coliform organisms to the upper respiratory tract, the incidence has been high in other series (Witkowski, 1935). These and other penicillin-resistant organisms are probably nearly always present and capable of contributing to the production of fatal lesions. It is not possible to say if penicillin has only eliminated penicillin-sensitive organisms to allow penicillin-resistant organisms to produce very similar lesions. In 1939 MacGregor emphasized the high incidence of $\mathbf{B}$. coli infections in neonatal pneumonia. While coliform organisms are only too easily isolated from the dead newborn infant it must be admitted that this organism, even in non-treated cases, often appears to be the only bacterial pathogen at the time of death. Occasionally in previous cases of this kind we have separated a few colonies of Staphylococcus aureus from a heavy growth of coliform organisms. In the present series the autopsy material was rarely fresh enough to permit satisfactory bacteriology, but no penicillin-sensitive organisms were recovered. In many cases of this kind in the newborn it is probable that the type of bacteria causing the pneumonia is less important than the conditions rendering the respiratory tract of the newborn abnormally susceptible to bacterial infection.

It should be emphasized that there is no panacea for neonatal sepsis. While penicillin may be highly beneficial in a few cases, where a penicillinsensitive organism can be solely incriminated, its widespread exhibition can only distract attention from such symptomatic treatment as the control of dehydration and the prevention of aspiration pneumonia by good nursing. Very extensive researches, and perhaps radical changes in our conceptions of the interaction of infecting agents with one another and with epithelial surfaces, must precede advances in the treatment of neonatal sepsis. Since chemotherapy cannot control or cure the more widespread and more infectious forms of neonatal sepsis, the proper policy is to emphasize the necessity for its prevention by continued improvement in the provision of adequate accommodation, facilities for segregation and rigid nursing technique.

\section{Summary}

A diagnosis of neonatal sepsis was made in seventy-one cases and every alternative case was treated with both penicillin and sulphadiazine. The remaining cases received sulphadiazine alone and served as controls. A study of the sixty-one cases which were suitable for analysis failed to show that penicillin increased the recovery rate, or influenced either the duration of the illness or the severity of decline in body weight. The importance in this connection of bacterial infection, especially of the respiratory tract by a potentially variable flora, is discussed. A few cases, chiefly of Staphylococcal infection, were thought to benefit in a further group of eighteen cases treated without controls.

The Belfast Penicillin Clinical Trials Committee is indebted to the Penicillin Trials Committee of the Medical Research. Council for supplies of penicillin. The authors are greatly indebted to Dr. Kathleen McKee for careful notes on the patients treated in the Royal Maternity Hospital; and to the nursing staffs of the respective hospitals.

\section{REFERENCES}

Kneeland, Y., Jr. (1930). J. Exp. Med., 51, 617.

MacGregor, A. R. (1939). Arch. Dis. Childh., 14, 323.

Morison, J. E. (1945). J. Path. Bact., 57, 221.

Torrey, J. C., and Reese, M. K. (1944). Amer. J. Dis. Child., 67, 89.

- (1945). Ibid., 69, 208.

Witkowski, R. (1935). Zentralbl. Bakt. (Abt 1), 133, 334. 